\section{The Olympus EVIS LUCERA Variable Indices of Haemoglobin Chart Function: a Novel Technique for Establishing the Completeness of Vascular Mucosal Ablation in Colonic Angiodysplasia}

\section{P. Hurlstone, M. Karageh, \\ D. S. Sanders}

Gastroenterology and Liver Unit, Royal Hallamshire Hospital, Sheffield, United Kingdom

\section{Acknowledgements}

Grant support for this publication was received from the Smith and Nephew Research Foundation, the BRET Research Foundation, a Butterfield award from the Great Britain Sasakawa Foundation, and the Mason Medical Research Foundation.

D. P. Hurlstone, M. D.

Room P39/Ward P2,

Gastroenterology and Liver Unit The Royal Hallamshire Hospital Glossop Road

Sheffield, South Yorkshire S10 2JF

United Kingdom

Fax: $\quad$ +44-114-271-2692

E-mail: p.hurlstone@shef.ac.uk

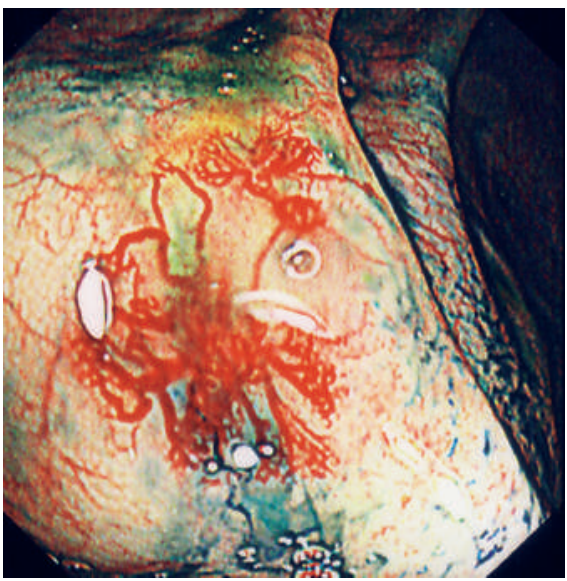

Figure 1 A 78-year-old man presented with recurrent iron-deficiency anaemia. Colonoscopy showed a vascular angiodysplastic lesion in the ascending colon.

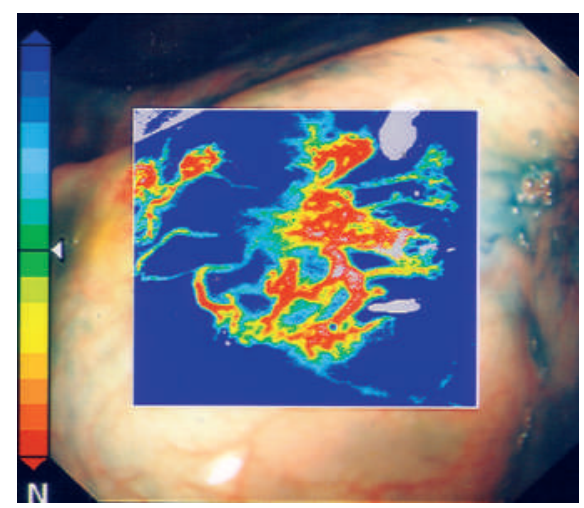

Figure 2 Using the indices of haemoglobin (IHb) colour chart function provided by the Olympus EVIS LUCERA system, the reference $\mathrm{IHb}$ values are displayed in yellow, with areas higher than the reference value (hypervascular) displayed in "warm" colours (i.e., orange-red) and hypoperfused areas displayed in "cool" colours (blue-grey). IHb charting of the lesion shows a clear vascular map. The lesion was ablated under $\mathrm{IHb}$ guidance using argon plasma coagulation (APC).

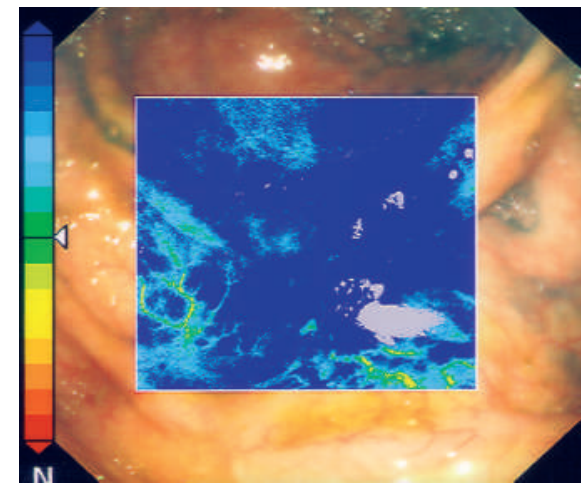

Figure 3 After APC ablation, IHb mapping was repeated. Hypoperfused mucosa with complete ablation of the vascular lesion is evident, as demonstrated by a completely blue $\mathrm{IHb}$ chart. 